\title{
Evaluation of Ethiopian Influenza Sentinel Surveillance System
}

\author{
Desalegn Takele ${ }^{1,}$, , Shikur Mohamed ${ }^{2}$ \\ ${ }^{1}$ Public Health Emergency Management, Ethiopian Public Health Institute, Addis Ababa, Ethiopia \\ ${ }^{2}$ Department of Public Health, Saint Paul's Hospital Millennium Medical College, Addis Ababa, Ethiopia
}

Email address:

desalegnpapa@gmail.com (D. Takele)

*Corresponding author

\section{To cite this article:}

Desalegn Takele, Shikur Mohamed. Evaluation of Ethiopian Influenza Sentinel Surveillance System. Automation, Control and Intelligent Systems. Vol. 9, No. 1, 2021, pp. 1-5. doi: 10.11648/j.acis.20210901.12

Received: November 30, 2020; Accepted: December 14, 2020; Published: January 22, 2021

\begin{abstract}
Background: Ethiopia conducts influenza sentinel surveillance since 2008 in eight sites through the coordination of Ethiopian Public Health Institute although little is known whether the system meets its objective. Hence, this evaluation is conducted to evaluate the sentinel surveillance attributes, purposes and its operation system. Method: A cross-sectional descriptive study was conducted from February 15-30, 2017 in all eight Sentinel sites. Data were collected using US-CDC updated surveillance guideline and Interview with influenza sentinel surveillance focal persons, regional public health emergency officers and national surveillance officers. Case based reports of influenza like illness and severe acute respiratory illness were also reviewed. Secondary data were collected from the national public health emergency management center based at EPHI. We analyzed and compiled the data. Results: Not all the visited health facilities have posted the ILI and SARI cases definition. None of the sentinel sites have been reporting influenza data to their next higher level but to the national PHEM (NIL). All focal persons have responded that they are expected to do so. Data is only being analyzed by national PHEM. Supportive supervision was done this month (February, 2017) since 2014. Laboratory feedback (test result) has been provided irregularly since May 2016 by the national influenza laboratory to sentinel sites and respective regional PHEM. All of focal persons have taken training on influenza surveillance. The positive predictive value (PPV) was $21.35 \%(\mathrm{n}=4922)$. Among a total of 5,097 case based reports from 2008-2016, $47(0.9 \%)$ age variable, $385(7.5 \%)$ temperature variable, and $103(2 \%)$ date of specimen collection were not filled. Conclusion: Although focal persons are satisfied with the forms and procedures involved, they are not filling formats properly as expected and reporting regularly as scheduled neither to the national PHEM nor to the next higher level. The influenza sentinel surveillance system has proven to be useful in providing virological data used to characterize and monitor influenza trends in Ethiopia. Continuous supportive supervision should be in placed using checklist to increase the quality of data. Data should be continuously analyzed and feedback should be given periodically to health care provider and partners.
\end{abstract}

Keywords: Surveillance, Evaluation, Influenza, Ethiopia

\section{Background}

Public health surveillance is an ongoing systematic collection analysis, interpretation and dissemination of data regarding a health related event for use in public health action to reduce morbidity and mortality and to improve health. A communicable disease surveillance system serves two key functions; early warning of potential threats to public health and programme monitoring functions which may be disease- specific or multi-disease in nature [1] Surveillance system evaluation allows us to define whether a specific system is useful for a particular public health initiative and is achieving the overarching goals of the public health program and the data collection objectives [2]. This is done for monitoring disease trends, describing natural history of diseases, identifying epidemics or new syndromes, monitoring changes in infectious agents, identifying areas for research so as eventually to reveal disease burdens and guiding the action to be taken, the health policy, planning, 
evaluation of health programs and the likes [3].

The Global Influenza Surveillance and Response System (GISRS), previously known as the Global Influenza Surveillance Network (GISN), has been performing influenza virological surveillance since 1952 [4].

In recent years, an increasing awareness has developed of the need to expand influenza surveillance and to include more epidemiological information to complement the virological data collected by GISRS. This need was formally recognized by the World Health Assembly in 2011 in Resolution 64.5 and in the adoption of the Pandemic Influenza Preparedness Framework [5].

The pandemic of 2009 uncovered several specific gaps in global influenza surveillance capacity, which compromised the assessment and monitoring of the event. The lack of a pre-existing international mechanism for sharing epidemiological data presented challenges to understanding global patterns of transmission and disease. And finally, the non-standardized approach to data collection and outbreak investigations early in the event resulted in data that was often incompletely understood outside the local context. The accumulation of historical data for influenza-associated severe respiratory disease will allow rapid comparative assessment of each influenza season and of future pandemics both locally and globally.

Public health surveillance systems play a critical role in national health management. They provide data that can be used for several purposes [6]. Data disseminated by a public health surveillance system can be used for immediate public health action, program planning and evaluation, and formulating research hypotheses [7]. For example, in America, Public health officials rely on the timeliness and completeness of the surveillance system data to design public policies and interventions [8].

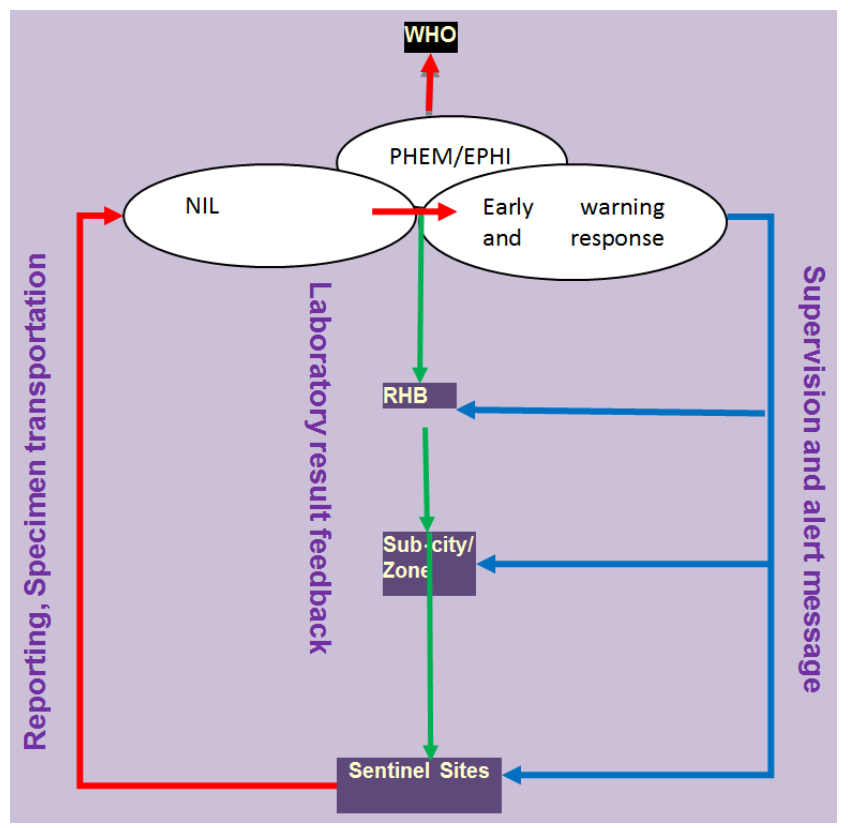

Figure 1. Ethiopian Influenza Sentinel surveillance Information Flow.

Ethiopia has launched influenza sentinel surveillance since
2008 [9] and currently five SARI and three ILI sites are engaged in the influenza surveillance. It is carried out by Federal Ministry of Health/EPHI through a system which has support from CDC and WHO and extending from EPHI down to the sentinel site. These includes upward and down ward reporting and feedback mechanism (Figure 1).

Sites were selected based on availability of resources, technical capacity and number of staffs, availability of functional service for ILI/SARI for all age groups, accessibility to adequate clients and geographical proximity between SARI and ILI sites.

\section{Rationale for Influenza Surveillance System Evaluation}

The world health organization has put a standard about periodic evaluation of influenza sentinel surveillance which states a comprehensive evaluation of the system to be done regularly, beginning one to two years after initial implementation of the surveillance system or more importantly if an expansion of the system is being considered [10]. However, since the start of sentinel surveillance the system, documents showing an accomplished evaluation of the system were not found except one that was done by FELTP resident in 2012 [11]. Little is known whether the system meets its objective. Hence, this evaluation was conducted to evaluate the sentinel surveillance attributes, purposes and its operation system

\section{Methods}

\subsection{Study Design and Area}

A cross-sectional descriptive study was conducted in February 2017 in all eight Sentinel sites. All influenza sentinel surveillance data from 2008-2016 was included in the study. Five SARI sites (Yekatit 12, Adama, Adare, Mekelle and Felegehiwot hospitals) and three ILI sites (Shiromeda, Akaki and Kolfe health centers) were included in the study.

\subsection{Data Source and Collection}

Primary data were collected using structured questionnaire and Interview with influenza sentinel surveillance focal persons, regional health bureau and national surveillance officers. Case based reports of influenza like illness and severe acute respiratory illness were also reviewed. Secondary data were collected from the national public health emergency management center based at Ethiopian public health institute.

Evaluation methods from Centers for Disease control and Prevention (CDC), Updated Guideline for Evaluating Public Health Surveillance Systems: Recommendation from the Guidelines working Group, MMWR July 27, 2001; 50 (No. RR-13) and Communicable disease surveillance and response systems, Guide to monitoring and evaluating, WHO, 2006 
were used to collect information about the surveillance system. Structured questionnaire was prepared and used to collect data about the core functions of surveillance, support functions of surveillance and attributes of surveillance system

\subsection{Data Analysis and Report Writing}

Data was analyzed and compiled using Microsoft excel.

\section{Results}

\subsection{Functions of Surveillance}

\subsubsection{Case Detection and Registration}

During this evaluation, not all the visited health facilities have posted the cases definition of ILI and SARI but the understanding of the cases definitions by the influenza focal persons was satisfactory. The registration book was found in all sites except Adama Teaching Hospital but the handling of available registers was poor in all sites.

\subsubsection{Reporting}

There was no shortage of reporting forms in all visited sentinel sites since it is also available in soft copies. But in Adare General Hospital and Adama Teaching Hospital, computer was not functional for the influenza surveillance data management that it was intended to. All sites use similar reporting format which is incorporated in the influenza sentinel surveillance implementation guideline. None of the sentinel sites have been reporting influenza data to their next higher level but to the national PHEM (NIL). All focal persons have responded that they were expected to do so. The weekly reporting rates of the sentinel sites over the past 12 weeks prior to assessment were 50\% (4/8). All the reports were sent to the National Influenza Laboratory in hard copies along with the throat swab samples.

\subsubsection{Data Analysis and Interpretation}

Although they have computer in all sentinel sites, except Adama and Adare hospitals, all influenza surveillance focal persons have never analyzed and interpreted their data routinely but only for presentations during review meetings; both case-based and aggregated. It is only analyzed by national PHEM. All of the focal persons have responded that they do not have basic computer skills.

\subsubsection{Feedback and Supervision}

Supervision and provision of feedback were assessed at national and regional levels. This assessment found that periodic supervision was not done. Supportive supervision regarding influenza surveillance was done this month (February, 2017) since 2014. Laboratory feedback (test result) has been provided irregularly since May 2016 by the national influenza laboratory to sentinel sites and respective regional PHEM.

\subsubsection{Training}

There are 14 influenza surveillance focal persons currently working in place. All of them have taken training on influenza surveillance. Including facility medical directors and regional PHEM officers, a total of 29 persons were trained on influenza surveillance in November, 2016.

\subsection{Surveillance System Attributes}

\subsubsection{Usefulness}

Before the beginning of influenza sentinel surveillance in Ethiopia nothing was known about influenza strains circulating in the country. Currently because of the surveillance data generated by the system, type of influenza strains, its burden and trends are being understood. A number of scientific papers were developed and presented at various national and international conferences. Sporadic pandemic influenza A (H1N1) in 2009 and seasonal influenza outbreaks in 2016 were detected by the surveillance and prompt response was instituted.

\subsubsection{Simplicity}

Participating health workers at sentinel sites were well aware of the objectives of the system. Collection of specimen and filling case based reporting format was found to be taking only 5-10 minutes. Case definition and reporting format is clear and easy to use by all level professionals. All sentinel sites focal persons were satisfied with reporting formats and easy flow of reports directly to national PHEM. The system has only few organizations involved in receiving case reports.

\subsubsection{Flexibility}

A flexible public health surveillance system can adapt to changing information needs or operating conditions with little additional time, personnel, or allocated funds. The system was found flexible; in 2009 the system was easily customized and detects pandemic influenza. The case definition by itself can also be used to detect any other respiratory infections like Middle East respiratory syndrome corona virus (MERS- CoV).

\subsubsection{Data Quality and Completeness}

Data quality reflects the completeness and validity of the data recorded in the public health surveillance system. In contrast to simplicity of the system, some important variables were in case based reports. For instance, among a total of 5,097 case based reports from 2008-2016, 47 (0.9\%) age variable, $385(7.5 \%)$ temperature variable, and $103(2 \%)$ date of specimen collection variables were not filled (Table 1).

Table 1. Number (percent) of variables missed during implementing influenza sentinel surveillance in Ethiopia, 2008-2016 ( $N=5097)$.

\begin{tabular}{lll}
\hline Variable & $\begin{array}{l}\text { Incomplete } \\
\text { data (\%) }\end{array}$ & $\begin{array}{l}\text { Above Expected percentage } \\
(\mathbf{8 0} \%)\end{array}$ \\
\hline Age & $47(0.92)$ & Yes \\
Zone & $182(3.57)$ & Yes \\
Woreda & $685(13.43)$ & Yes \\
Body Temperature & $385(7.55)$ & Yes \\
Antiviral & $557(10.93)$ & Yes \\
Date Specimen Collection & $103(2.02)$ & Yes \\
Date Sent to lab & $2,267(44.48)$ & No \\
Specimen Condition & $181(3.55)$ & Yes \\
\hline
\end{tabular}




\subsubsection{Acceptability}

All level health personnel, especially sentinel site focal persons are happy to participate in the surveillance system. The laboratory workers in NIL have taken self-initiatives to transport specimens to laboratory from all sentinel sites of Addis Ababa twice a week. Six sentinel sites (except Adama and Adare hospitals) out of eight have dedicated a room for SARI/ILI surveillance purpose and all eight sites have assigned trained focal persons.

\subsubsection{Sensitivity}

Influenza like illness and severe acute respiratory illness outbreaks were detected in 2016 using the ILI and SARI case definitions, and prevention and control measures were taken. In this evaluation the denominator was not available to calculate sensitivity of the surveillance system. However, beside loose of case definition which is very sensitive to ILI/SARI cases, specimen tested at EPHI laboratory was also tested positive at WHO reference laboratory.

\subsubsection{Predictive Value Positive}

The positive predictive value (PPV) or the proportion of cases reported by the system that actually have influenza was $21.35 \%$ $(n=4,922)$. The capacity of the laboratory diagnostic test was periodically ensured by WHO confirmatory laboratory.

\subsubsection{Representativeness}

The ILI/SARI Sentinel surveillance is limited to only four regions and one city administration, Addis Ababa. Even though the sites were selected to represent most of the population, it is still difficult to generalize to the whole population. In the system the catchment population was not clearly defined; because of this, calculating the incidence rate was difficult.

\subsubsection{Timeliness}

Timeliness in this report reflects the elapse between date of symptom onset and date of specimen collection. In this regards, the range of the elapsed time is between two and seven days which is exactly in line with the time indicated in the Ethiopian national influenza sentinel surveillance implementation guideline.

\section{Discussion}

Generally, this evaluation revealed that the Ethiopian influenza sentinel surveillance is on the right track to meet intended objectives. This sentinel surveillance had identified circulating influenza viruses in Ethiopia which are pandemic influenza A (H1N1) 2009, Seasonal influenza A (H1N1), Seasonal influenza A (H3N2) and influenza B. Seasonality and trends are also defined as in October, November and December [12]. The season is not very different from Albanian influenza season [13].

Regarding the functions of surveillance system, there are gaps in reporting, data analysis and interpretation because of lack of skill from the sentinel site focal persons side. Failure to conduct periodic supportive supervision from the national and regional side might have bolded this gap. As it was stated in the influenza implementation guideline, monthly supportive supervision for sites of Addis Ababa and quarterly for others had to be done by the national surveillance/national influenza laboratory and periodic supervision and feedback were expected to be performed by regional/zonal/woreda/sub-city offices [9]. The sentinel surveillance system was found very simple; surveillance focal persons at sentinel site identify eligible cases, collect throat swab samples with case based reporting format and the laboratory personnel from national influenza laboratory directly communicates with the sentinel site. This system is very easy for health care providers at sentinel site like Madagascar's influenza system [14].

The system has good data quality but there is a $44.48 \%$ incomplete variable (Date specimen sent to lab) which is almost half of the total case based forms. This shows focal persons at facility level have limitations in understanding or practicing the necessity of each variable in the form. Unless this field is filled, they cannot track storage condition of the specimen.

The sentinel sites do not represent the whole population. Even the catchment population was not clearly defined. Hence to calculate incidence rate and to generalize to the whole population, the sentinel sites will need to be more expanded considering Varity of geographic location and community life style. Lack of dedicated resources from government may affect the stability of influenza sentinel surveillance. The sentinel surveillance is donor dependent; this may affect sustainability of the system. All running cost, supplies and logistics are donated by US-CDC.

\section{Conclusion}

The influenza sentinel surveillance system has proven to be useful in providing virological and epidemiological data used to characterize and monitor influenza trends in Ethiopia. Generally, the staff members using the system are satisfied with the forms and procedures involved, but not all sites are reporting regularly neither to the national PHEM nor to the next higher level. The influenza sentinel surveillance system is simple, acceptable, stable, flexible and able to detect outbreaks, identify and characterize circulating influenza strains but yet to expand nationwide. Data quality is yet to be maintained.

\section{Recommendation}

Trainings should be given for sentinel site focal persons on reporting, data use and advantages of a complete data. Continuous supportive supervision should be in placed using checklist to increase the quality of data. Data should be continuously analyzed and feedback should be given periodically to health care provider and partners. Monitoring should be done as scheduled in the national influenza surveillance guideline to improve data quality. 


\section{Authors Contribution}

The work was carried out in collaboration between both authors. Desalegn Belay collected data, analyzed the data and wrote the manuscript. Shikur Mohammed advised and reviewed the manuscript.

\section{Conflicts of Interest}

Both authors declared they have no conflict of interest.

\section{Finance}

This work was done by the full financial support of the Ethiopian Public Health Institute.

\section{Acknowledgements}

We would like to thank Influenza sentinel surveillance focal persons from all eight sites for their cooperation during data collection. We also thank public health emergency management center and St Paul's Millennium medical college for facilitating all necessary logistics and advisory for the field work.

\section{References}

[1] WHO. Communicable disease surveillance and response systems. 2006. 1 p.

[2] CDC. Overview of Evaluating Surveillance Systems. 2013. 2 p.

[3] (CDC) C for DC and P. Updated Guidelines for Evaluating Public Health Surveillance Systems Recommendations. Vol. $50.2001 .3 \mathrm{p}$.

[4] WHO. Interim Global Epidemiological Surveillance Standards for Influenza [Internet]. 2012. 1 p. Available from:
http://www.who.int/influenza/resources/documents/INFSURV MANUAL.pdf?ua $=1$

[5] World Health Assembly S. Pandemic influenza preparedness : sharing of influenza viruses and access to vaccines and other benefits. In 2011. p. 3-4.

[6] Ngugi BK. evaluating the quality and usefulness of data from current hiv / aids surveillance systems in usa methodology / approach. 2009: 1-13.

[7] CDC. CDC surveillance evaluation. 2001. 1-35 p.

[8] Fahey RL, Fahey R. Evaluation of the System Attributes of Timeliness and Completeness of the West Virginia Electronic Disease Surveillance System â $€^{\mathrm{TM}}$ NationalEDSS Based System This is to certify that the doctoral dissertation by. 2015;

[9] FMoH E. Influenza Sentinel Surveillance,. 2012. 3 p.

[10] World Health Organization. Global Epidemiological Surveillance Standards for Influenza. Igarss 2014. 2014. 54 p.

[11] Abyot--An Evaluation of Ethiopian Influenza like Illness. 2012.

[12] Woyessa AB, Belay D alegn, Mengesha M, Zemelak E, Beyene B, Kasa W, et al. Epidemiology of Influenza in Ethiopia: Findings from Influenza Sentinel Surveillance and Respiratory Infection Outbreaks Investigation, 2009-2015. Vol. 891. 2015.

[13] Daouda Coulibaly, Hervé KADJO, Anderson K. N'gattia, Abdoulaye OUATTARA, Djibril Cherif, Kouakou K. Bertin, JP Yao Kouamé NSD. OPTIONS IX for Influenza. OPTIONS IX Influ. 2016; (August): 270.

[14] Randrianasolo L, Guillebaud J, Razanajatovo N, Andriamampionona L, Tempia S, Piola $\mathrm{P}$, et al. Evaluation of the Influenza Sentinel Surveillance System in Madagascar, • Influenza sentinel surveillance has been established. In 2014. p. $1-26$. 